\title{
Sachregister zu Band 68
}

Die fett gedruckten Zahlen bezeichnen Eigenarbeiten. Bb. = Buchbesprechung.

A

Abduzenslähmung nach Grippe 361

- $\quad$ isolierte - nach Schädeltrauma

183 Abführmittel, Schädigung des

Auges durch Einnehmen von -

101 Ablatio retinae 287, 288, 313

hämorrhagische 96

und ophthalmoskopische Topo-graphie 44

durch tumorartige Aderhaut-tuberkulose 102

Heilung der - mittels Ignipunktur des Netzhautrisses 313

Achsenbezeichnung, Vereinheit-

lichung der - 48 Achsensucher 50 Adenoidektomie beim Trachom 56 Aderhaut s. Chorioidea

Adrenalin, Wirkung des - auf den

intraokularen Druck 76 Afenil bei Frühjahrskatarrh 76 Agraffes, Nützlichkeit der - bei

der Vernähung des unteren Lides

179 Akkommodationsmesser 49 Akustisch-chromatische Syn-

opsien 194 Amaurose, hysterische 65 Anästhesie, experimentelle - der

Augenmuskeln 106

- regionäre - 200Anästhesierung des Trigeminus-

gebietes 372 Angioma capillare -hypertro-

phicum der Hornhaut 67 Angiomatosis retinae 207 Anisokorie, physiologische 63, 70 Anthrax

palpebrarum 311 Apex ossis petrosi, das Syndrom

des - 60 Applanationsprinzip der Tono-

metrie im Lichte manometrischer

Untersuchungen 46 Arteria centralis retinae, Ver-

schluß der - 303, 312

- $\quad$ Astverschluß der -108

Embolie eines Zweiges der - nach Injektion von Metarseno-benzol 121

das ophthalmoskopische Bild und die Sehfunktion bei Verschluß der - 82

Arteriosclerosis retinae, oph-

thalmologisch nachweisbare -

202 Astigmatismus, neue subjektive

Untersuchungsmethoden bei -48 Atheromatose derKarotis und der

Arteria ophthalmica, endokra-

nische Läsioned des Sehnerven

infolge von - 182 Augendruck s. Druck Augenerkrankungen, infektiöse

310 Augenheilkunde 190 (Bb.) Augenhintergrund bei Herzkran-

ken 202 Augenlider s. Lider Augenmedien, spezifisches Ge- 
wicht der - 288 Augenmuskeln, experimentelle

Anästhesie der - 106 Augenmuskellähmungen, ange-

borene 107 - Diagnostik der - 52 Augenpoliklinik des Allukraini-

schen Instituts für Hygiene und

Erziehung 310 Augenspiegel, reflexloser stereo-

skopischer Hand- - 47 Autohämatotherapie 74, 75, 78 Automobilfahrer, Sehvorschrif-

ten für - 47 Autovakzinebehandlung beiTra-

chom und anderen Conjunc-

tivitiden 79 Axenfeld-KrukenbergschePig-

mentspindel 282 Axenfeldsches Verfahren, Mo-

difikation des - bei Ausschnei-

dung der unteren Tränendrüse 39

B

Bacterium fluorescens liqu., In-fektion mit - 278

Basedowsche_Krankheit, Lid-schluß- undÖffnungsfunktion und Exophthalmus bei - 300

Beleuchtung, Bedeutung der - für Gesundheit und Leistungs-fähigkeit 378

Bestrahlung, Kammerwasserunter-suchungen nach -- 108

Biedl-Bardetsches Syndrom235

Bienenstiche, Iritis und Conjunctivitis nach -- 188

Sachregister zu Band 68.

383

Bindehaut s. Conjunctiva Binokulares Sehen, Prüfung des

- 100Blepharophimose, inkongruente

seitliche Verschiebung der Augen-lider bei seniler - 273 Blepharoptosis, bulbopalpebrales

hyperkinetisch.es Phänomen bei

- 189

Blickfeld, Gerät zur graphischen

Darstellung des - 345 Blickfeldtuch, Heßsches 308 Blindenwesen i22ff. Blindenwohlfahrt, Kongreß für

$-\quad 122$

Blitzkatarakt, Pathogenese der-

72 Blitzschlagverletzung 99 Blutdrucksteigerung, ein Zei-

chen allgemeiner - an den Netz-

hautgefäßen 113 Bluttransfusion, Netzhautblutun-

gen nach - 287 Blutung aus dem Conjunctivalsack

eines Neugeborenen 197 Blutverlust, Sehstörung durch -

195

Brechungsfehler, gutachtliche Be-urteilung von - im Bergbau 100

Brücke, Gliom der - 59

Bügel, seniler nicht myopischer -288

Bulbopalpebrales hyperkineti-sches Phänomen bei Blepharoptosis 189

C Canaliculus lacrimalis, Granu-lom nach einem Fremdkörper des

- 65Chalazion 273Chiasma,Gumma des - 62

- $\quad$ heteronyme binasale Hemianop-sie durch Läsion des - 56

Cholesterinkristalle in einer ka- 
taraktösen Linse 72 Chorioidea, Kolobom der - mit

Netzhautzysten 302

Geschwülste der - 286, 287

Krebsmetastase der -307

Tuberkulose der -68

Netzhautablösung durch tumor-artige Tuberkulose der - 102

- $\quad$ zirkumskripte Melanose der - 70Chorioiditis sympathica, Sensi

bilisierung bei - 300 Conjunctiva als Eingangspforte infektiöser Prozesse 289, 294

Diphtherie der -311

$\Lambda$ lelanosis der -108

Tuberkulose der -308

Conjunctivalsack, Blutung aus dem - bei einem Neugeborenen 197

- $\quad$ Plastik des - 199Conjunctivitis nach Bienenstichen

188

- $\quad$ Schwimmbad- - 274, 275-m syphilitische 276

- Autovakzinebehandlung der -

79

Vakzinebehandlung der Diplo-bazillen-C. 78

Cornea, Endothel der - 116

- $\quad$ erbliche Degeneration der - no-- Dystrophie der - 100

- $\quad$ Erkrankungen der - 65 ff., 281,282

- rezidivierende Erosion der -278

Pigmentierung der - bei Zigeu-nern 366

Verätzung der -276

Herabsetzung der Sensibilität der - durch Drechslerarbeit 277

- Behandlung von Krankheitender - mit ultraviolettem Licht152

Corneatrübung, gürtelförmige 306

- familiäre knötchenförmige - 349Corodenin, Verwendung des - bei

den industriellen Lichtquellen 89

Dakryocanaliculitis cystica 274 Dakryops 274

Descemetozelen, das Auftreten persistierender - 22

- $\quad$ pulsierende 280Diphtherie der Conjunctiva 311Diplobazillen-Protease 274Diplopie, monokulare - bei einem

Hysteriker nach Trauma 185 Druck, intraokularer, optische Registrierung des - 46

- Wirkung des Adrenalins auf den

$-\quad 76$

Dystrophia cachectico-genita

lis, Augenbefunde bei - 108 Dystrophia corneae 100

E Echinokokkus der Augenhöhle 364 Einschlußblennorrhoe 88 Eisensplitter im Auge, die Leistungsgrenzen beim Nachweis von

- 43

Eisensplitterextraktion 301 Eisensplitterverletzungen, Au-

tohämatotherapie bei - 74 Eiterherde, Krankheitserscheinun-

gen am Auge bei - an anderen

Teilen des Körpers 120 Ektopia lentis 119

$25^{*}$ 
$3 \S 4$

Sachregister zu Band 68.

Encephalitis lethargica, Augen-hintergrundsveränderungen bei

- 63

Endotheliom der Sehnervenschei-

den 183 Entropium spasticum des Ober-

lides 273 Epibulbäre Knötchenbildung

mit pseudotuberkulösem Typus67 Episkleritis 377 Epithelkörperchen, akzessorische

- $\quad$ bei der Ratte 285Ethmoiditis, chronische eitrige -

mit okularer Symptomatologie 62 Euphorbia Helioscopa, Ver

ätzung der Bindehaut und Horn-

haut durch - 276 Exkavation des Sehnerven bei Hy-

pophysistumor 194 Exophthalmus, angeborener -

mit Retractio bulbi $366-$ Messung des -50

die Diagnostik des inflammatori-schen - bei akuten Infektions-krankheiten 1

bei Basedow 300

-- bei Mucocele sinus ethmoidalis 199

$\Gamma$

Farbenblindheit, ungewöhnliche Form totaler - 107

Farbensinnprüfung 52

Farbige, insbesondere grüne Gläser als Augenschutz 205 (Bb.)

Fascia lata, Verwendung der - am Auge 27

Fettröpfchen in der Vorderhammer nach perforierender Ver-letzung 282

Fibrom der Cornea 68

Filaria loa, aus dem Unterlid ent-fernt 273

Focal sepsis 120

Fremdkörperextraktion 299,301

Fremdkörperlokalisation 115

- radiologische 121Friedmannsches Tuberkulose-

heilmittel 291 Frühj ahrskatarrh, Afeml bei - 76

G

Gefäßneubildung, syphilitische -

von der Papule in den Glas-

körper 306 Gehirn, Veränderungen des Auges

bei Erkrankungen des - 57 Gesichtsempfindungen 206 (Bb.) Gesichtsfeldaufnahmen, Verein-

heitlichung der - 48 Gesichtsfelduntersuchung 51

- $\quad$ nach Bjerrum 345

Gewerbliche Augenschädigun-

gen 378 Glasbläser, Ablösung der Zonula-

lamelle bei -284

- $\quad$ Kataraktbildung nach Uitravio-lettbestrahlung bei -295

Glaskörper, Strukturdes-285, 286

- Veränderungen der Vorderkam-mertiefe infolge veränderten In-haltes des - 188

- Niederschläge auf der Vorder-fläche des - bei Keratitis tuber-culosa 103

Glaskörperblutungen, Augen-hintergrund bei rezidivierenden 
- $\quad 3{ }^{\circ}$ Glaskörpertrübung, juvenile

94 Glassorten, Wirkung verschiede-

ner - auf das Auge 295 Glaukom 94, 112, 298

und Naevus flammeus 244

mit doppelseitiger Sehnerven-atrophie nach Schädelbruch 101

nach Venenthrombose 373

Möglichkeit der Beeinflussung des

- $\quad$ durch Röntgenbestrahlung derSchilddrüse 163

- Gynergen bei - 203Glaukomoperation, Kataraktent-

stehung nach -309

- $\quad$ basale Iridektomie 310Gliom des Mittelhirns und der

Brücke 59 Glioma retinae, hereditäres 303

- $\quad$ Behandlung des - mit Röntgen-strahlen 76, 78

Gol dchloridtätowage der Horn-

haut 191 Gonitis serosa bei Keratitis pa-

renchymatosa 64 Gonoblennorrhoe, Reizkörper-

therapie der - 276 Gradenigosches Syndrom 60 Granulom nacheinem Fremdkörper

des Canaliculus lacrimalis 65 Grippe, Lähmung des Abduzens

nach -361

- Neuritis optica nach - 199Gumma chiasmatis 62Gynergen bei Glaucoma simplex

203

H Halskompression, Augenverände-

rungen bei isolierter traumati-

scher - 65 Hämangiom der Orbita 102

der Meningen mit Beteiligung des Sehzentrums 315

zerebrales - mit kontralateraler Hemiplegie bei Naevus angio-matosus der Haut 313

Sachregister zu Band 68.

385

Handaugenspiegel, reflexloser

stereoskopischer - 47 Hebelnystagmographie 52 Heliotherapie, künstliche - bei

Trachom 75, 77 Hemianopsie, heteronyme bina-

sale - durch Läsion des Chiasma

und der Sella turcica 56

- $\quad$ linksseitige homonyme - mitErhaltenbleiben des temporalenHalbmondes 190

-- periphere und zentrale - 374 Herpes corneae 277, 278 Herpesvirus, Ophthalmia sympa-

thica durch das - 291, 292 Herxheimer-Reaktion am Lim-

bus corneae 278 Herzkranke, Augenhintergrundbei

- $\quad$ 202Heterochromie 283, 284Hornhaut s. CorneaHydrophthalmus und Entwick-

lungsstörung der Iris 115

- $\quad$ und Naevus flammeus 85Hypercholesterinämie und Xan-

thomatosis bulbi 281 Hypertension des Liquor, Ver-

halten des hinteren Labyrinths

bei experimenteller - 58 Hypophysin, Einfluß des - auf

die Pupille 188 Hypophysisextrakt, Wirkungdes

- $\quad$ auf das Auge 186Hvpophysistumoren, Diagnose 
und Therapie der -317

- $\quad$ Sehnervenschädigung durch -

97. J94 Hypopyonkeratitis 68

- $\quad$ Stomosine bei - 76, 78Hypotonie, akute - bei Ablatio

retinae 288

- $\quad$ künstliche - des Bulbus an Ver-suchstieren 117

Hysterische Amaurose 65

I

Infektionskrankheiten, die Dia-gnostik des inflammatorischen Exophthalmus bei akuten - 1

Instrumente, Apparat zur Pruning der Schärfe von Operations-118

Iontophorese 74, 75, 118

Iridektomie, basale - bei Glau-kom 310

Iridoziliare Venen, im ganzen Verlauf sichtbare - 283

Iridozyclitis, eitrige - bei kon-genitaler Lues 283

Iris, Hydrophthalmus und Ent- $\Lambda$ vicklungsstörung der - 115

- $\quad$ luetische Entzündungen der -71

Iris, traumatische Motilitätsstö-

rungen der - 184 Iriszyste 104 Iritis nach Bienenstichen 188

E

Kalkablagerung im Auge, eine besondere Art der - 221

Kalkverätzung des Auges 277

Kammerwasseruntersuchung 286

- nach Bestrahlung 108

Kampimeter 51

Krebsmetastase der Chorioidea

$3^{\circ} 7$

im orbitalen Abschnitt des Seh-nerven 371

Katarakt 71, 72, 284, 285, 294,

295

Ringstar 372

Entstehung der - nach Glau-komoperation 309

-- Entstehung des Altersstars 284

Gewichtsverhältnisse seniler - no

die renale Funktion bei -60

einseitige Schmelzhypoplasie bei einseitiger Zonular- - 196

degenerative Hornhautverände-rung bei Myotonie- - 282

Zahnveränderungen bei Zonular-- 285

Iontophorese in der Therapie der -74

Kataraktoperation 122

der gegenwärtige Standpunkt der Alters star extraction in der Kap-sel in, 369

mit einem Brückenlappen nach Vaschez-Denis 118

bei einer Patientin mit Polyarthritis ancylotica 118

Keratitis 279

bullosa haemorrhagica 67

disciformis 67

neuroparalytica nach Schädel-trauma 183 
neurotrophica superficialis 278

parenchymatosa 308

- Gonitis serosa bei - 64

- Einfluß der Salvarsanbehand-lung der Eltern auf die Häufig-keit der - 280

profunda, histologischer Befund bei -260

tuberculosa und Lichen scrofulo-sorum 280

- Niederschläge auf der Vorder-fläche des Glaskörpers bei - 103

Keratocon j unctivitis lympha-tica 61

385

Sachregister zu Band 68.

Keratohypopyon 68

- Stomosine bei - 76, 78

Keratokonus endokrin. Ursprungs

durch Opotherapie geheilt 376 Keratoplastik, penetrierende no Knötchenbildung, epibulbäre mit pseudotuberkulösem Typus 67 Kollargol 279 Kontaktgläser 86, 87 Konvergenzreaktion der Pupille

und Naheinstellung 119 Korektopie, Kasuistik der - 20

L

Labyrinth, Verhalten des hinteren

- $\quad$ bei experimenteller Hyper-tonie des Liquors 58

Lawrence-Biedlsches Syndrom

302 Lichen scrofulosorum und Ke-

ratitis tuberculosa 280 Lichtbehandlung des Auges 296 Li der, inkongruente seitliche Ver-

schiebung der - bei seniler Ble-

pharophimose 273 Liderkrankung nach Teakholz-

verarbeitung 309 Lidkolobom, angeborenes 304 Lidplastik, Nützlichkeit der

,, Agraffes" bei der Vernähung

des unteren Lides 179 Lidspalte, abnorme Länge der -

84 Lidtumoren 371 Lidwinkel, abnorme Gestalt des

äußeren - 84 Limbus corneae, Herxheimer-

Reaktion am - 278 Linse, experimentelle Untersuchun-

gen der -112

Einfluß der Röntgenstrahlen auf die embryonale - 304

Erkrankungen der $-71,72$

Blutung in die -372

Häutchenbildung auf der vorde-ren Kapsel der - 284

in toto verkalkte -308

in vivo nachgewiesene verkalkte

$-\quad 30$

- $\quad$ Wanderung der luxierten - unterdie Conjunctiva 184

Liquor cerebrospinalis bei $\mathrm{Au}-$

generkrankungen 63 Lochbildung in der Makulagegend

37i Lues, luet. Entzündungen der Iris

und des Corpus ciliare 71 Lungentuberkulose, Augenver-

änderungen bei - 61 Lysolverätzung des Auges 276 
$\mathrm{M}$

Macula lutea, die richtige Bestim-

mung der - 51 [ Maculadegeneration, familiäre

progressive - 289 I Maculagegend, Lochbildung in der

I -371

Makropsie, physikalische Theorie der - 189 Malaria, Augenkomplikationen bei

- 55 Massagekatarakt 72 Melanodermie bei melanotischem

Skleraltumor 185 Melanosarkom, extrabulbäres -

der Orbita 200 Melanose, zirkumskripte - der

Chorioidea 70 Melanosis conjunctivae 108

- retinae 80

Membrana uvealis, angiokrino-pathische Entzündungen der - 187

Meningitis serosa, Augensym-ptome bei -58

- plötzliche Erblindung durch-57Metarsenobenzol, Embolie eines

Zweiges der Zentralarterie nach

Injektion von - 121 Mikropsie, physikalische Theorie

der - 189 Mikroskopie des lebenden Auges

379 (Bb.) Mikuliczsche Krankheit 371

- $\quad$ Augenerkrankungen bei - 274Mittelhirn, ,Gliom des - 59Morphium, Einfluß des - auf

das

Auge 187 Mucocele des Sinus ethmoidalis,

Exophthalmus bei - 199 Musculus obliquus superior,

traumatische Lähmung des - 183 Muskelanomalien, angeborene

Ptosis und - 315 Myiasis 353 Myopie, Genese der - 194

- $\quad$ hochgradige - und künstlicheUnterbrechung der Schwanger-schaft 127

Myotoniekatarakt, degenerative Hornhautveränderung bei - 282

$\mathrm{N}$

Nachstarmesser 372

Naevus angiomatosus der Haut mit zerebralem Hämangiom und kontralateraler Hemiplegie 313

- $\quad$ flammeus und Glaukom 244

- $\quad$ - und Hydrophthalmus 85Naheinstellung, Konvergenzreaktion der Pupille und - 119

Sachregister zu Band 68'

387

Narbenaufhellungsstreifen,

Fuchssche - 281 Nasenschleimhaut, Erscheinun-

gen der - beim Trachom 56 Nephritis, Neuroretinitis bei - 80 Nervenerkrankungen, Verände-

rungen des Auges bei - 57 Nervocidin, Schmerzstillung durch

$-367 \mathrm{Ne}$ Vvus oculomotorius, zyklische

Lähmung des - 95 Nervus opticus, Erkrankungen

des $-181,182$

Schädigung des - durch Hypo-physistumor 97

-Atrophie, doppelseitige mit Glaucoma simplex nach Schädel-bruch 101

- bei Pseudotabes 59

- - nach Stauungspapille bei Turmschädel 192

Tumoren der scheiden 375 
Netzhaut s. Retina Netzhautablösung s. Ablatio

retinaeNeuritis op tic a nach Grippe 199 durch Vergiftung mit Thallium-

azetat 203 Neuroretinitis bei Nephritis 80 Neurosis ciliaris vasomotorica

dolorosa bilateralis mit Hy-

potonie des Bulbus 187 Nieren bei Kataraktösen 60 Noma der Orbita 293 Nystagmus, Nachweis

von schwa-

chem -52

- optiko-kinetischer 55

- $\quad$ nystagm. Reaktionen in einemFall von multipler Sklerose 55

0

Oguchische Krankheit 289 Ohrerscheinungen bei Ophthalmia sympathica 56 Okulo-

hypophysäres Syndrom nach Enchondrom des Sinus sphenoidalis 61 Omnadin 297

Ophthalmia metastatica 293 Ophthalmia sympathica 84, 291, 292

aurikuläre Erscheinungen bei der - 56

durch das Herpesvirus 291, 292

Heilung einer - nach inter-kurrentem Scharlach 291

Ophthalmologie, über den Fort-schritt in der - und ihre künf- i tigen Errungenschaften 33

Ophthalmomyiasis interna an-terior 353

Ophthalmoplegia externa pro-

gressiva chronica 95 Opsonogeninj ektionen, Rezidiv eines Herpes corneae nach - 277 Optiker, die deutschen - 298 Orbita, Hämangiom der - 102 Orbitaltumor, bestrahlter 198 - extrabulbäres Melanosarkom 200

$\mathrm{P}$

Pankreasdiabetes, ophthalmolo-gische Studien bei experimen-tellem - 283

Pannus, Denigsche Tran splanta-tion zur Behandlung des - 356

Parietallappenabszeß, syndrom-gekreuzte Lähmung nach oto-genem - 57

Periostitis alveolaris, Orbital-phlegmone nach - 294

Periphlebitis retinae nontuber-culosa 288

Phlegmone der Orbita nach Periostitis alveolaris 294

P.hotographie des Auges 43

Photother apie beim Trachom

75, 77 Plasmazellen, mehrkernige - und

das besondere Vorkommen von

Russellscher > Körperchen 134 Plastik des Conj unctivalsackes 199 Polyarthritis ancylotica, Kataraktextraktion bei einer Pa-

tientin mit - 118 Polycythaemiavera,Augenverän-

derungen bei - 308 Proteinkörperinjektionen in

den Glaskörper 291 Pseudoglioma retinae 82 Pseudotabes mit Sehnervenatro-

phie 59 Pseudotumor orbitae 309 Pyozyaneus-Infektion 278 Pseudozyste im Limbus 281

Pterygium 280 Ptosis, angeborene - und Muskel-

anomalien 315 Ptosisoperation nach Blaskovics

98 Pupillenbahnen 184 Pupillendistanzmesser 50 Pupillenreaktion, Konvergenz-

reaktion und Naheinstellung 119 Pupillensaumfilz 284

$\mathrm{R}$

Rachenschleimhaut, Erschei-nungen der - beim Trachom 56

Radiologische Studien über kra-niopharyngeale Tumoren 55 
Radiumbehandlung, endosphe-noidale - 77

388

Sachregister zu Band 68.

Reizkörpertherapie 297

- der Gonoblenorrhoe 276Rekurrenserkrankungendes Au-

ges 289 Retina, Erkrankungen $\mathrm{d}^{\wedge} \mathrm{r}-79 \mathrm{ff}$.

exsudative Erkrankungen der -201

ein Zeichen allgemeiner Blut-drucksteigerung an den Gefäßen der - 113

ein neuer Weg zur Lokalisation von Stellen der - 45

scheibenförmige Entartung der -mitte 315

- zystische Entartung der Peripherie der - 331

familiäre Zysten der - bei Ader-hautkolobom 302

degeneration mit multiplen Aneurysmen 207

Retinitis, das Krankheitsbild der

- 207

circinata bei Vaquezscher Krankheit 64

exsudativa 198

pigmentosa bei familiärer Taub-heit und Demenz 59

proliferans 316

Retractio bulbi bei angeborenem

Exophthalmus 366 Ringabszeßbildung 278 Ringskotom bei Affektionen der

inneren Membranen des Auges

und des Sehnerven 189 Rinnenbildung, periphere- der

Hornhaut 281 Röntgenaufnahmen, skelettfreie-

des vorderen Bulbusabschnittes 44 Röntgenstrahlen, Einfluß der -

auf die embryonale Linse 304

- $\quad$ Behandlung des Glioma retinaemit - 76, 78

Russellsche Körperchen, mehr-kernige Plasmazellen und das besondere Vorkommen von - 134

$\mathrm{S}$

Salvarsan, Einfluß der Behandlung der Eltern mit - auf die Häufigkeit der Keratitis paren-

chymatosa 280

Sarkome des Auges 287

- glioblastisches - des Stirn-lappens 63

Schädelbruch, doppelseitige Seh-nervenatrophie mit Glaukom nach

- $\quad$ 101Schädelmißbildungen, Läsionen

des Sehnerven bei - 182 Schädeltrauma, Abduzensläh-mung und Keratitis neuropara-lytica nach

$-183$

Scharlach, Heilung einer sympa-thischen Ophthalmie nach inter-kurrentem 291

Scheitelbrechwertmessung.48 Schielen, Ätiologie des - 104

Behandlung des - 198

Dosierung in der chirurgischen Behandlung des -180

stereoskopische Bilder für schie-lende Kinder 51, 199

Schieloperationen 105, 299 Schielwinkel, Gerät zur Messu ng

des - 345 Schilddrüse, Möglichkeit der Be-

einflussung des Glaukoms durch 
Röntgenbestrahlung der - 163 Schmelzhypoplasie bei einseitiger

Zonularkatarakt 196 Schmerzhaftigkeit bei Augen-

leiden 42 Schneeblindheit 294 Schutzgläser mit dünnen Metall-

schichten 295 Schwi mm bad-Conjunctivitis

274. 275 Schwangerschaftsunterbre-

chung, künstliche - und hoch-

gradige Myopie 127 Sella turcica, heteronyme binasale

Hemianopsie durch Läsion der -

56 Sensibilisierung bei Chorioiditis

sympathica 300 Siderosis corneae 277 Sinusaffektionen, Erkrankungen

des Sehnerven bei - 181 Sinus sphenoidalis, okulo-hypo-

physäres Syndrom nach Enchon-

drom des -61

- $\quad$ endosphenoidale Radiumbehand-lung nach operierten Läsionendes - 77

Skiaskopie 48

Sklera, experimentelle Untersu-chungen über die - 282

Kollaps der -185

melanotischer. Tumor der - mit Melanodermie 185

Skleritis 377

- $\quad$ und Amotio mitPigmentierung 302Sklerokeratitis tuberculosa,

Trauma als Ursache einer - 280

Sklerose, multiple, nystagmische

Reaktionen in einem Fall von -

55 Sklerosis orbitae 293 Solitärtuberkel der Chorioidea286 Status thymicolymphaticus in

Verbindung mit doppelseitigem

Kortikalstar 285 Stauungspapille, Entstehung der

$-372$

Sachregister

zu Band 68.

389

Stauungspapille, Sehnerven-atrophie nach - bei Turm-schädel 192

Stirnlappen, Tumoren des - 62,

63 Stomosine bei Keratohypopyon

und Conjunctivitis blennorrha-

gica 76, 78 Strabismus s. Schielen Strahlenbehandlung 296 Strahlenkatarakt 294

Syndesmoplastik 179 Synopsien, akustisch-chromatische

194 Syphilis, syph. Gefäßneubildung

von der Papule in den Glas-

körper 306 Syphilom, geheiltes des Ziliar-

körpers 283

$\mathrm{T}$

Targesin 279

Tätowierung der Hornhaut 191,192

Taubheit, familiäre - mit Erbl· in-

dung und Demenz 59 Teakholzverarbeitung, Lid- 
erkrankung nach - 309 Tebeprotin 290

Thalami optici , Funktion der-61 Thalliumazetat, Neuritis optica

durch Vergiftung mit - 203 Tintenstiftverletzung 370 Tonometer, Kontrolle des Schiötz-

schen - 45 Tonometrie, das Applanations-

prinzip der - im Lichte manome-

trischer Untersuchungen 46 Topographie, ophthalmoskopische

- und Netzhautablösung 44 Tournayscher Reflex 63, 70 Trachom 275, 276

Adenoidektomie beim - 56

Autohämatotherapie bei -74 ,

75,78

Autovakzinebehandlung des -79

Künstliche Heliotherapie bei -

$-75.77$

Behandlung des - mit Ionto-phorese 118

Erscheinungen der Nasen- und Rachenschleimhaut beim - 56

Beziehungen zwischen Tuber-kulose und - 57

Tränendrüse, Modifikation des - Axenfeldschen Verfahrens bei der Ausschneidung der unteren 39

Tränenwege, Erkrankungen der - 274

Transplantation, Denigsche zur Pannus-Therapie 356

Traubenzuckerinjektionen in den Glaskörper 291

Traumatologie $183 \mathrm{ff}$. Trigeminusgebiet, Anästhesie-

rung des - 372 Trypaflavin 98 Tuberkelbazillen, Eindringen der

- durch den unbeschädigten

Konj unktivalsack 311 Tuberkulose des Auges 290, 291,

293 - der Bindehaut 308

der Chorioidea 68

Netzhautablösung durch tumor-artige - der Chorioidea 102

Beziehungen zwischen Trachom und -57

Tumoren des Frontallappens, pu-pillare Symptome bei - 59

- $\quad$ radiologische Studien über kra-niopharyngeale - 55

Turin schädel, Sehnervenatrophie nach Stauungspapille bei - 192

Tutokain 180

Tyrosinkristalle in einer katarak-tösen Linse 72

U Ulcus corneae, Lichtbehandlung

des - 296 Ultramikroskopie des lebenden

Auges 43 Ultrarote und ultraviolette

Strahlen, Einfluß hochkonzen-

trierter - auf das Auge 96 Ultraviolette Strahlen in der

okularen Therapie 79

Behandlung von Hornhauterkran-kungen mit - 152

Kataraktbildung nach - bei Glasbläsern 295

Unfallkunde 18311., 204, 377 Uveitis chronica anterior 70

V Vakzinebehandlung der Diplo-bazillenconj unctivitis 78

- $\quad$ bei Orbitalentzündungen 297Vaquezsche Krankheit, Retinitis

circinata bei - 64 Verätzung des Auges 276, 277 
- $\quad$ Transplantation der Mundschleim-

haut bei - 377 Verkupferung des Auges durch

intraokuläre Kupfersplitter 289 Versicherungskunde 204, 377 Vorderkammer, Fettröpfchen in

der - nach perforierender Ver-

letzung 282 -- Veränderungen der Tiefe der -

infolge veränderten Glaskörper-

inhaltes 188

$39^{\circ}$

Sachregister zu Band 68.

W

Wärmeanwendung amfreien Auge

297 Wilsonsche Krankheit, Limbus-

pigmentierung bei - 281 Wismutin den Geweben und Säften

des Auges 73 Wortblindheit, kongenitale 307

$\mathrm{X}$

Xanthomatosis bulbi und Hyper-

cholesterinämie 281 Xeroderma pigmentosum 197

Zahnveränderungen bei Zonular-

katarakt 285 Ziliarkörper, luetische Entzün-

dungen des -71

Ziliarkörper, traumatische Moti-

litätsstörungen des - 184 Ziliarneuralgien als Prodrome des

Glaukoms 373 Zonularkatarakt, einseitige

Schmelzhypoplasie bei einseitiger

- 196 Zonulalamelle, Ablösung der -

284 Zyklodialyse, histologische Be-

funde bei -71

- $\quad$ Spätresultate der - 114Zykloiridoplegie, traumatische

184 Zysten der Hornhaut 281

- der Retina, erworbene - 80

- $\quad$ epidermoidale- der infundibulo-hypophysären Region 62

Zysticercus endocularis 82

Abe 274, 291 Abelsdorff 96, 371,

373 Accardi 73, 186 Addario La Ferla 55,

183, 187 Adrogue 316, 375 Alaj mo 72, 187, 379 Aliquò-Mazzei 183 Alexander 372 Altland 98, 102 Altschul 115 Amsler 44 Angelucci 74, 75 Apin 46 Arganaraz 377 Arnold 50 Aron 106, 107 Ascher 104, 105, 117,

118 Aust 87, 88, 192 Awguschewitsch 364

B

Bachstez 51 Back 278 Balod 281 Barczinski 353 Bartels 100, 102 Baumert 301 Baurmann 285

Berger 67, 277 Berneaud 48 Bertolotti 55 Beselin 306 Best 104, 107, 115 Bielschowsky 105, 106

Namenregister.

Birch-Hirschfeld 
122, 127, 296 Blatt 377 Block 378 Boente 95 Borowski 370 Borello 80 van den Bosch 103 Bosse 279 Bossuet 278 Braun 107, no, 203,

297 Brock 371 Brunetti 55 Bucklers 96, 97, 194, 372 Busacca 72 Busacca Gorgone 56

$\mathrm{C}$

Calogero 56 Carmi 75 Carot 203 Carrari 57

Cattaneo 66, 68, 70, 80 Cavaniglia 76 Cholina 119, 310 Chramelow 311 Chronis 297 Cirincione 316 (P). Coenen 300 Colden 107 Comberg 43, 45, 108,

194. 195. 37i Cords 297 Cozzoli 179

Currado 188 Cushman 315 Czellitzer 195

\section{$\mathrm{D}$}

Daniels 308 Danilewsky 199, 200 Denti 66 Derkac 281 De Rosa 57,9 70, 7 ,188 Deutsch 87

Deutschmann 287 Di Fede 57, 76 Di Giuseppe 188 Di Marzio 57, 58, 70 76, Dinger 276, 277

Dubois 44

E Eidelberg 119 Einar 202 Elschnig 105, no, iiï,

1. 112, 284, 287 Engelking 294 Eppenstein 373 Epstein 311, 371 Erben 117 Erlanger 370, 371 Esser 276

F Falta 42, 43 Farina 189 Favaloro 66, 71, 179 F aza kas 3S6 Federici 76, 184 Feeder 197 Fehr 97 